\title{
annie
}

\section{in 10 minutes}

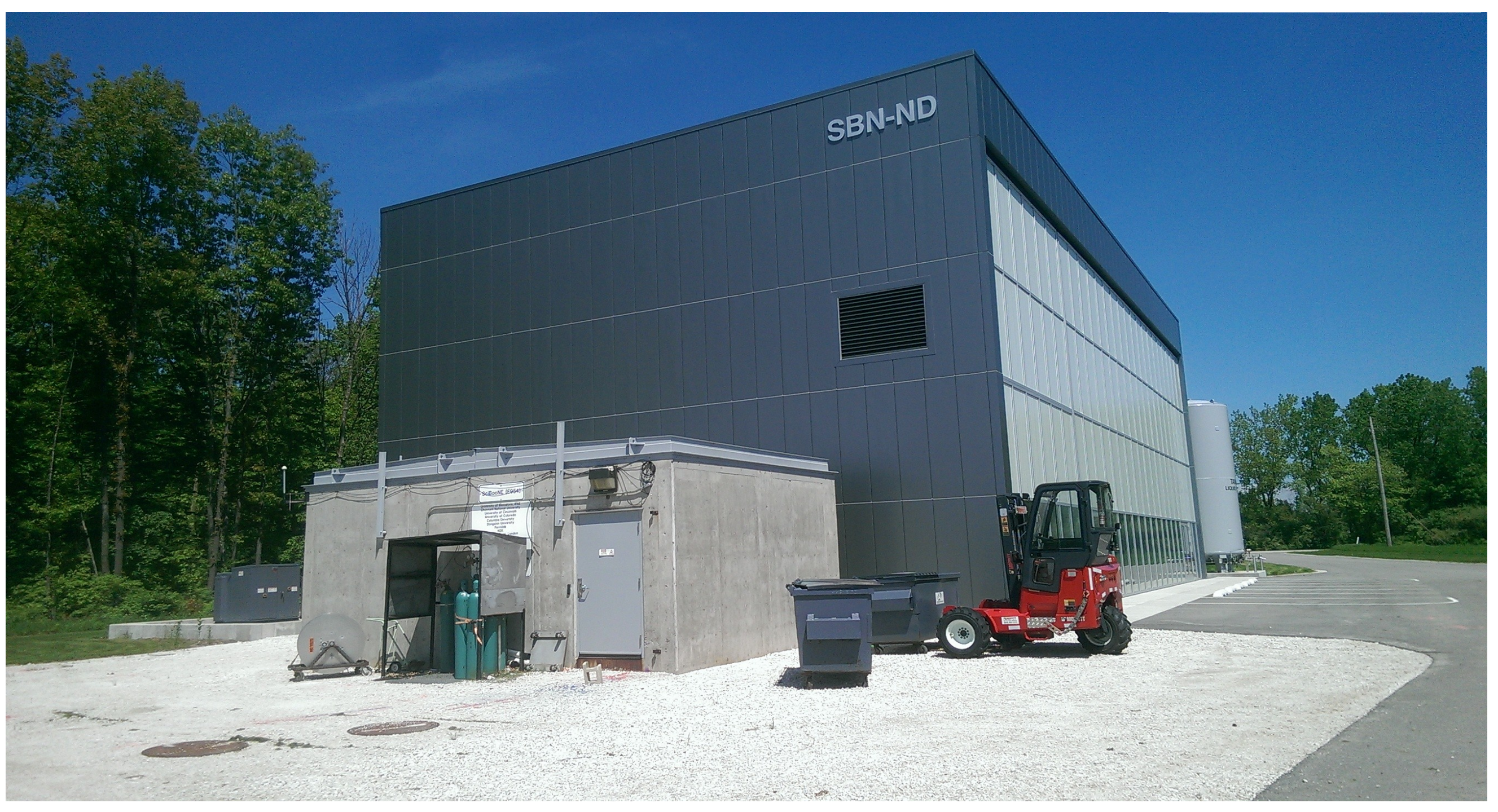

This document was prepared by [ANNIE Collaboration] using the resources of the Fermi National Accelerator Laboratory (Fermilab), a U.S. Department of Energy, Office of Science, HEP User Facility. Fermilab is managed by Fermi Research Alliance, LLC (FRA), acting under Contract No. DE-AC02-07CH11359. 


\section{True CCQE}

- 2 body scattering

- Target nucleon assumed at rest

- Kinematics from observed lepton
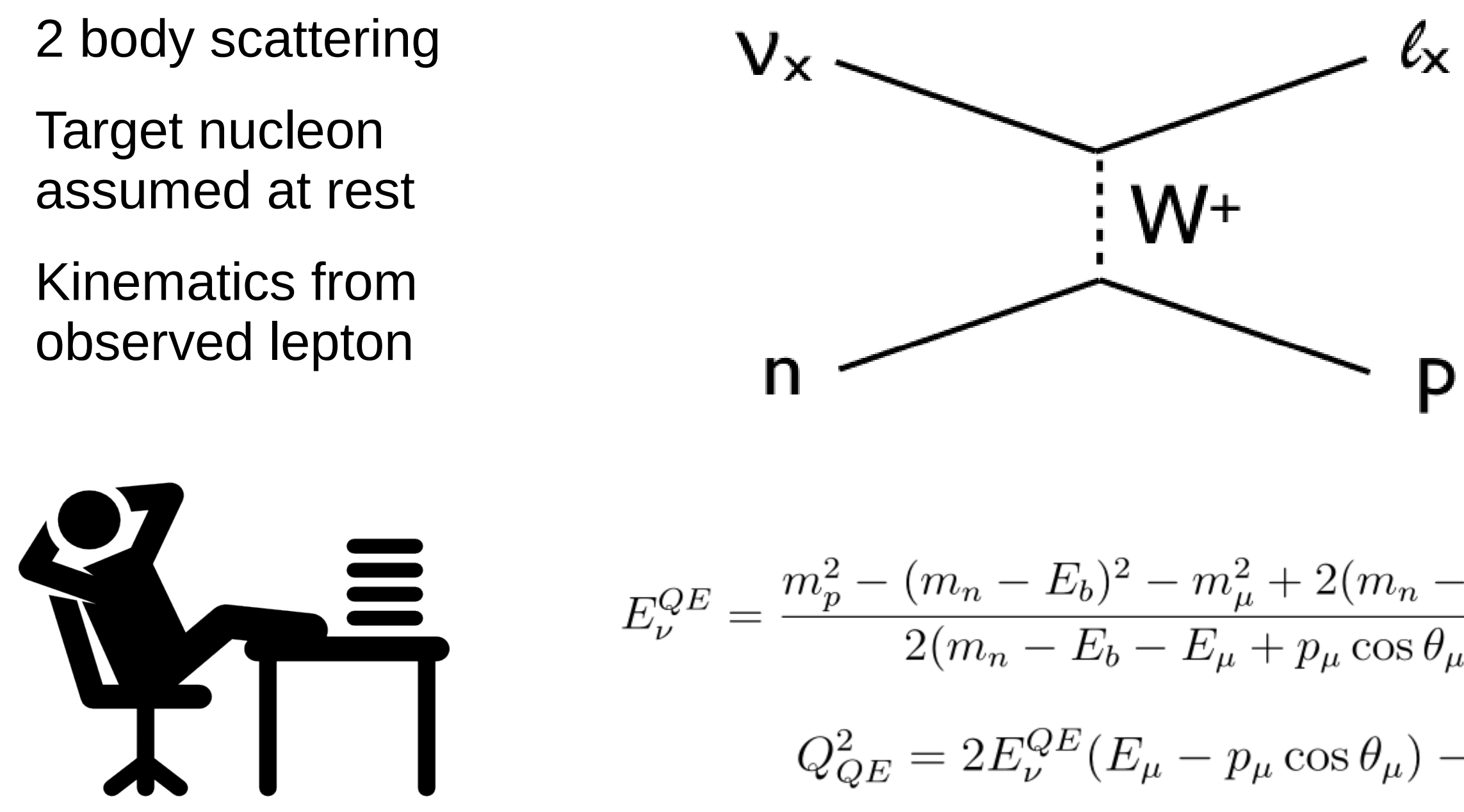

$$
\begin{gathered}
E_{\nu}^{Q E}=\frac{m_{p}^{2}-\left(m_{n}-E_{b}\right)^{2}-m_{\mu}^{2}+2\left(m_{n}-E_{b}\right) E_{\mu}}{2\left(m_{n}-E_{b}-E_{\mu}+p_{\mu} \cos \theta_{\mu}\right)} \\
Q_{Q E}^{2}=2 E_{\nu}^{Q E}\left(E_{\mu}-p_{\mu} \cos \theta_{\mu}\right)-m_{\mu}^{2}
\end{gathered}
$$




\section{Fake CCQE}

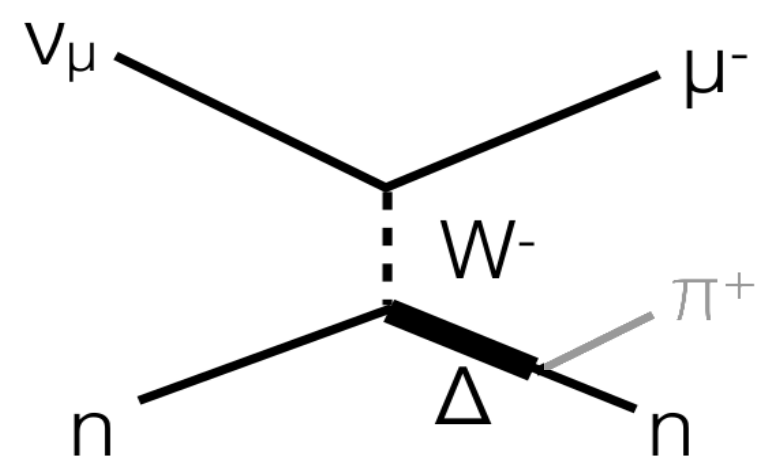

Resonant pion production with undetected pion

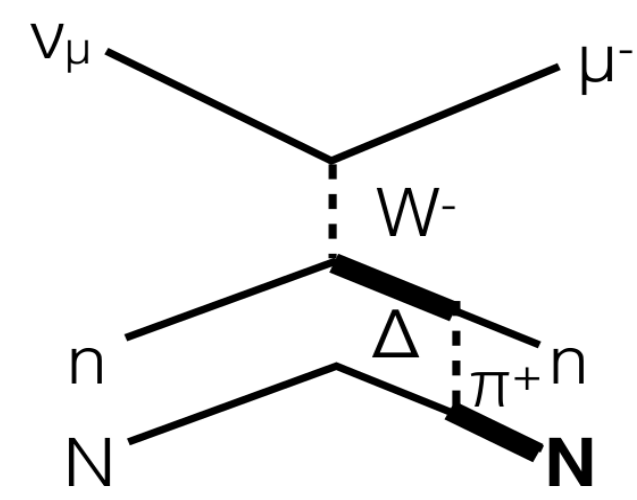

Resonant pion production with 'stuck pion'

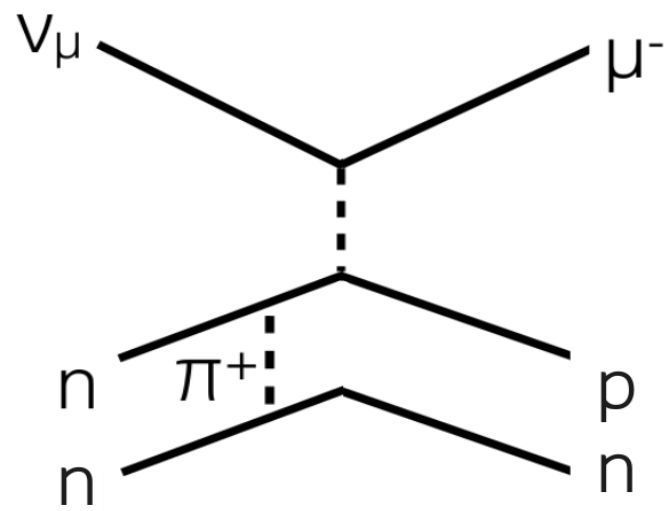

Interaction with correlated pair of nucleons

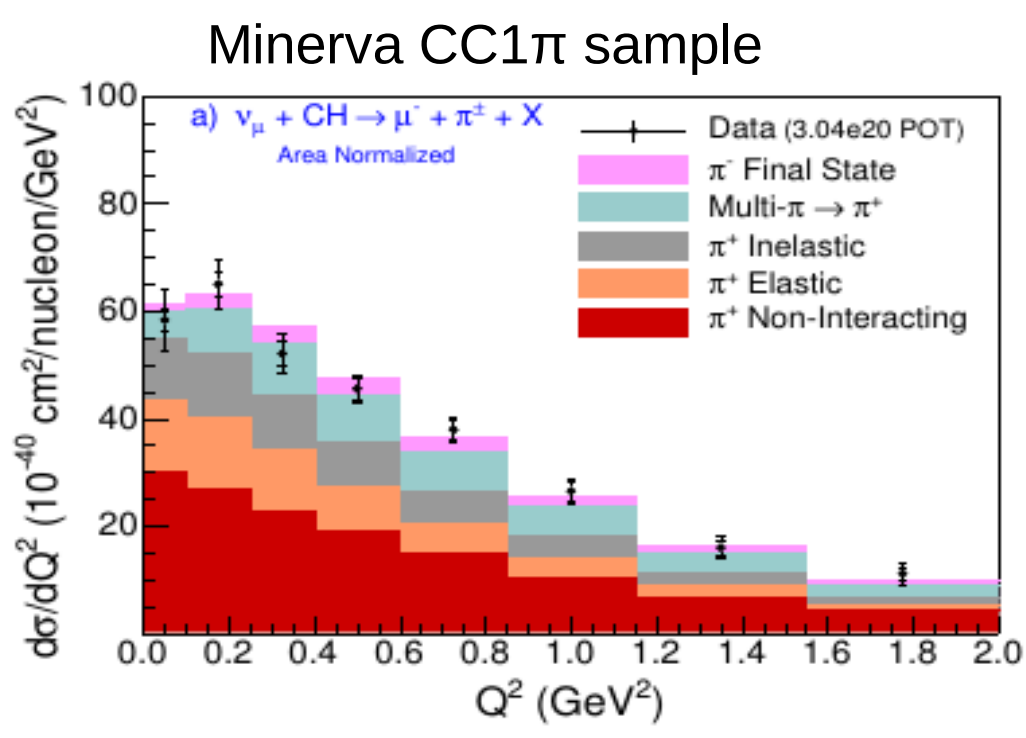

Phys.Rev. D94 (2016) no.5, 052005

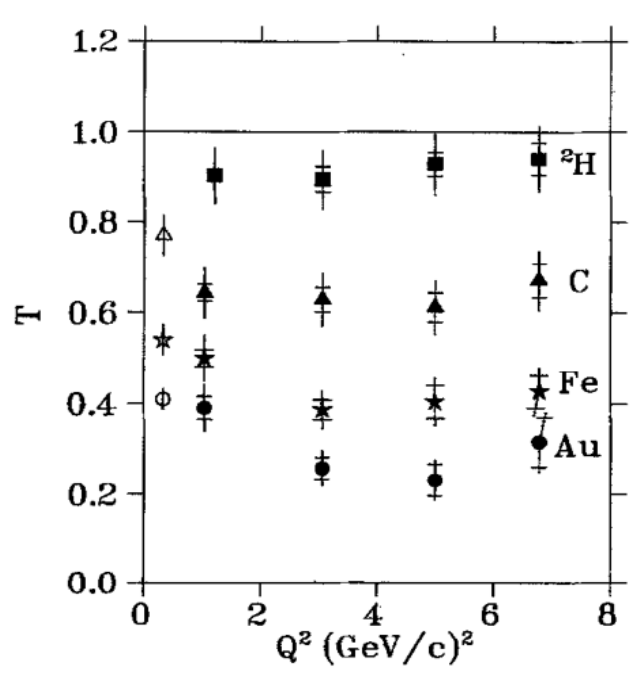

Phys. Lett. B 351 87-92
Fraction of protons that escape without re-interaction in e-p elastic scattering 


\section{Physics Impacts}

- Downward bias of reconstructed neutrino energy

- Influences measurement of oscillation parameters

- Source of IBD background

- Relevant for PDK and DSNB detection

- Efficiency of neutron tagging in excluding these interactions must be well known

- Accounting for these affects requires knowledge of multiplicity and kinematic relations
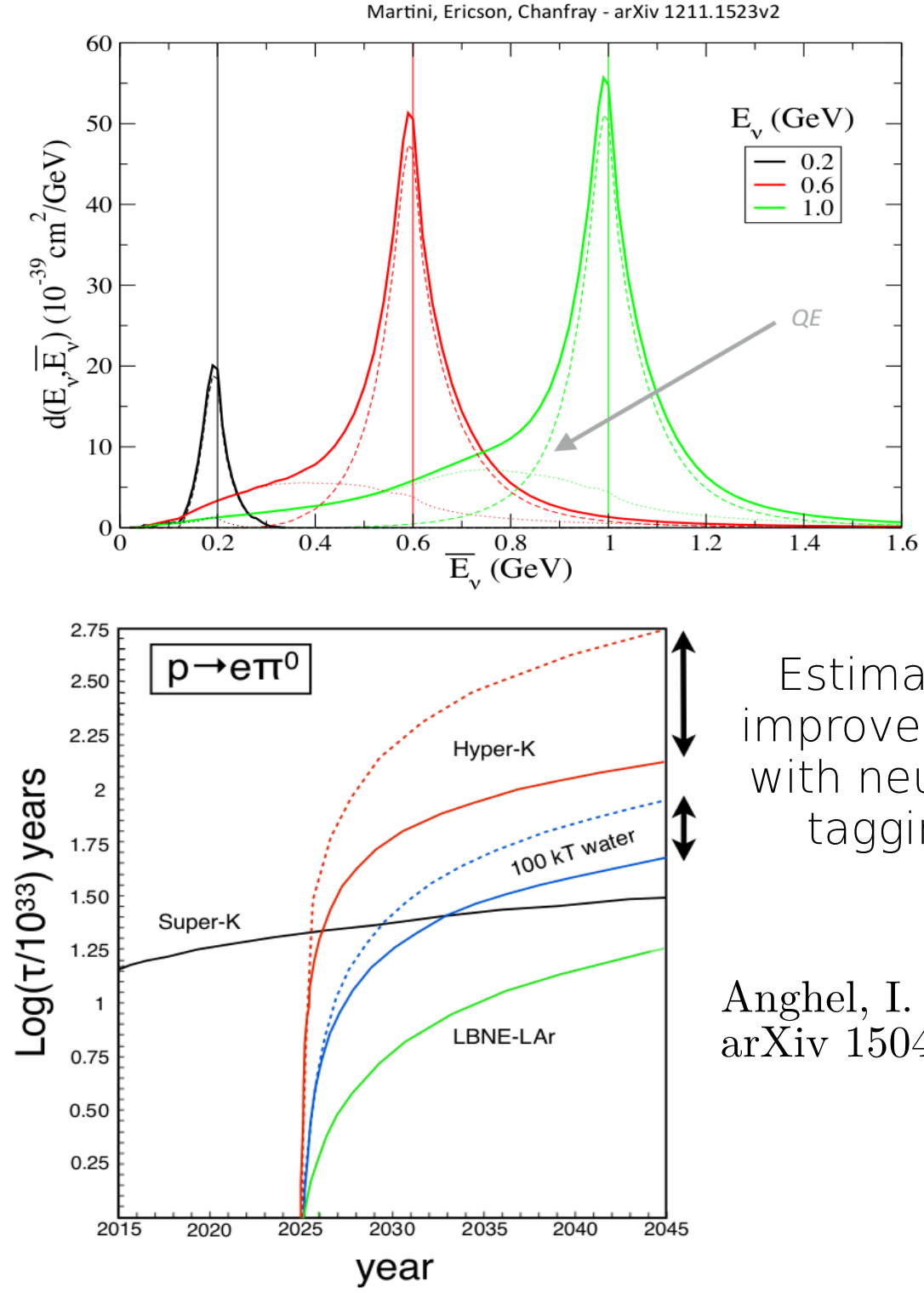

Estimated

improvement

with neutron

tagging

Anghel, I. et al., arXiv 1504.01480 


\section{Liquid Argon Measurements}

- High resolution and low threshold of LAr experiments make protons visible down to $21 \mathrm{MeV}$

- Multiplicity measurements by ArgoNeut demonstrate discrepancies between generators and data

- Experimental input is crucial to improve generators and theory!
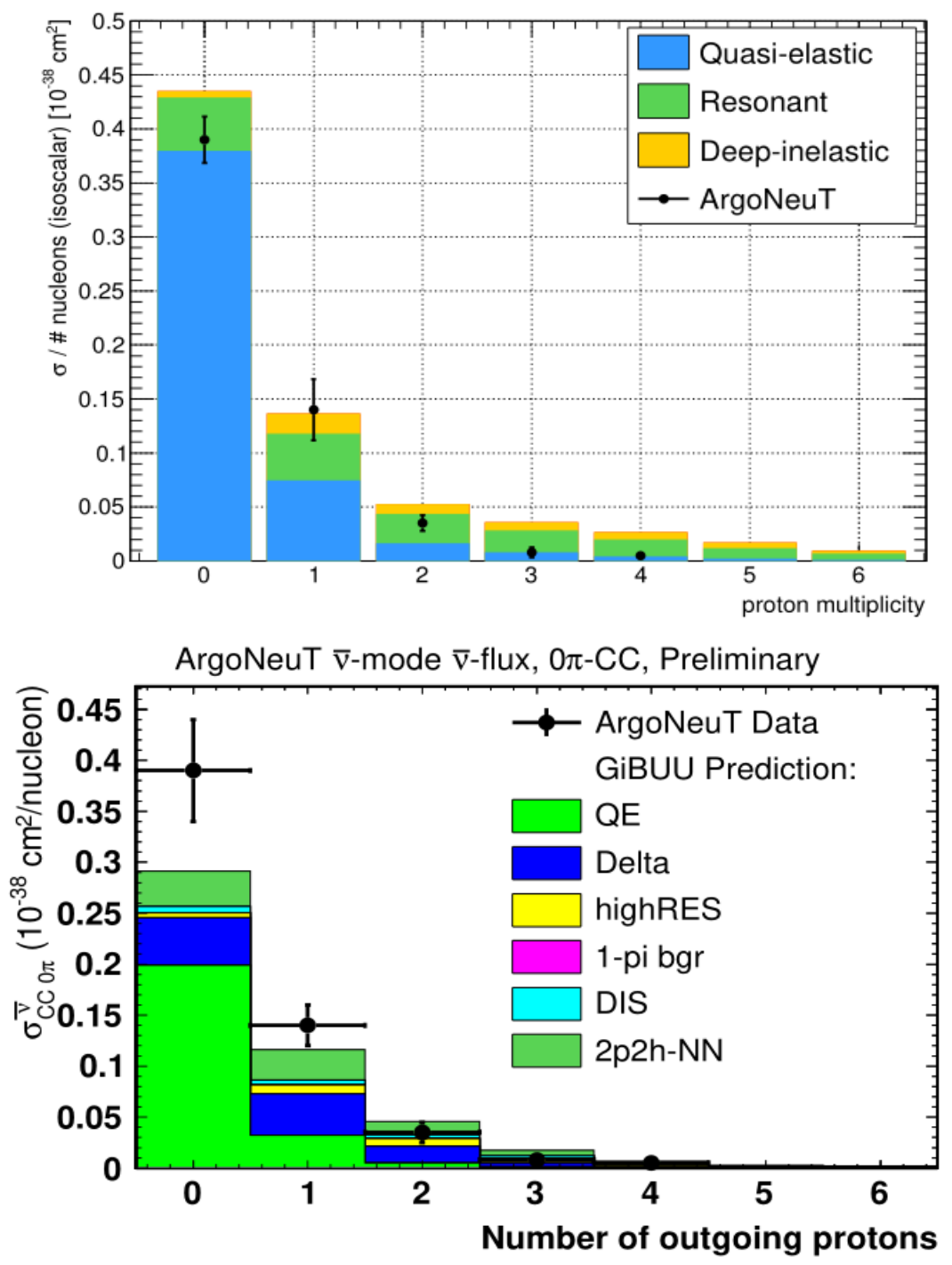

https://doi.org/10.7566/JPSCP.12.010017 


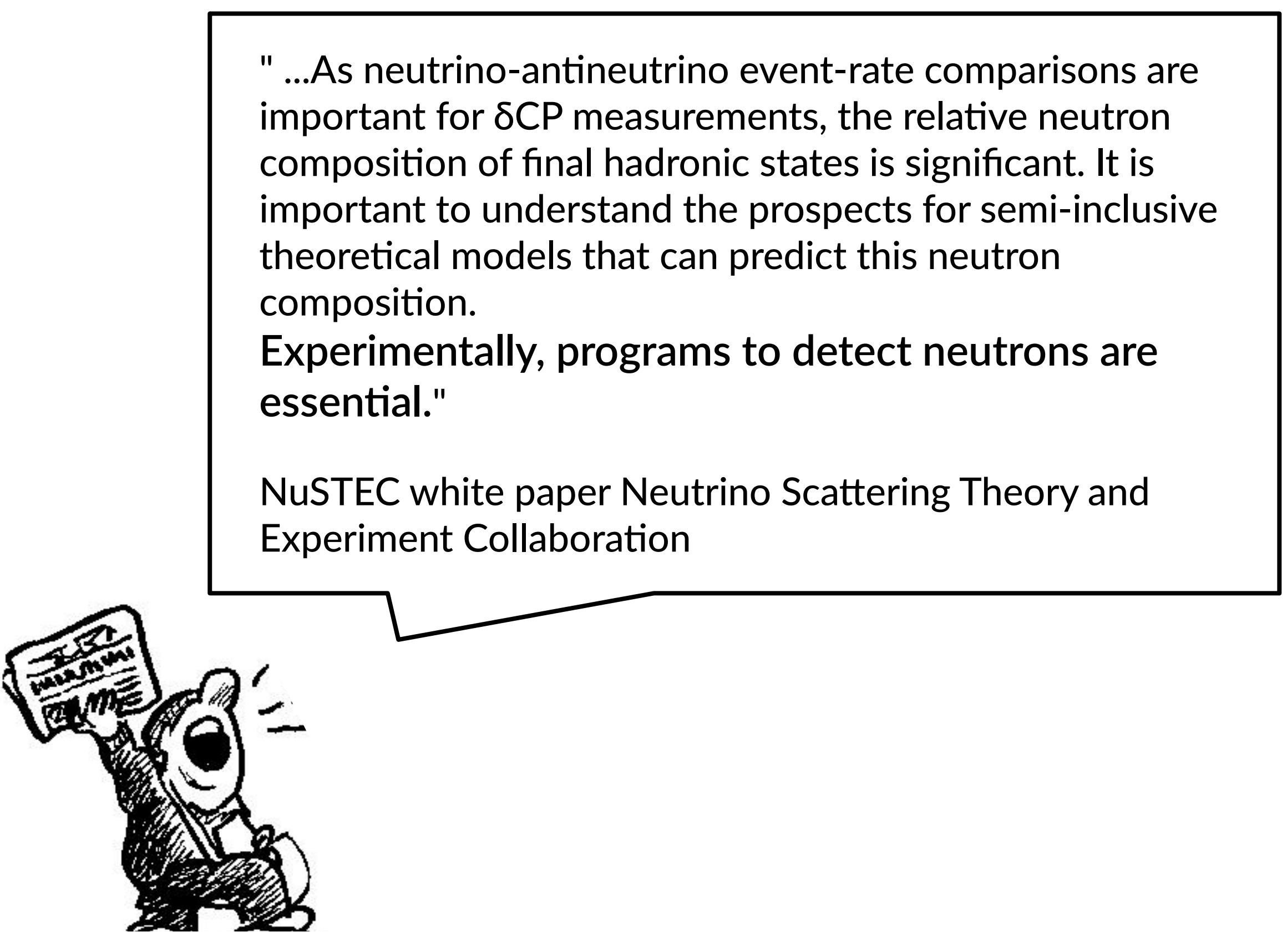




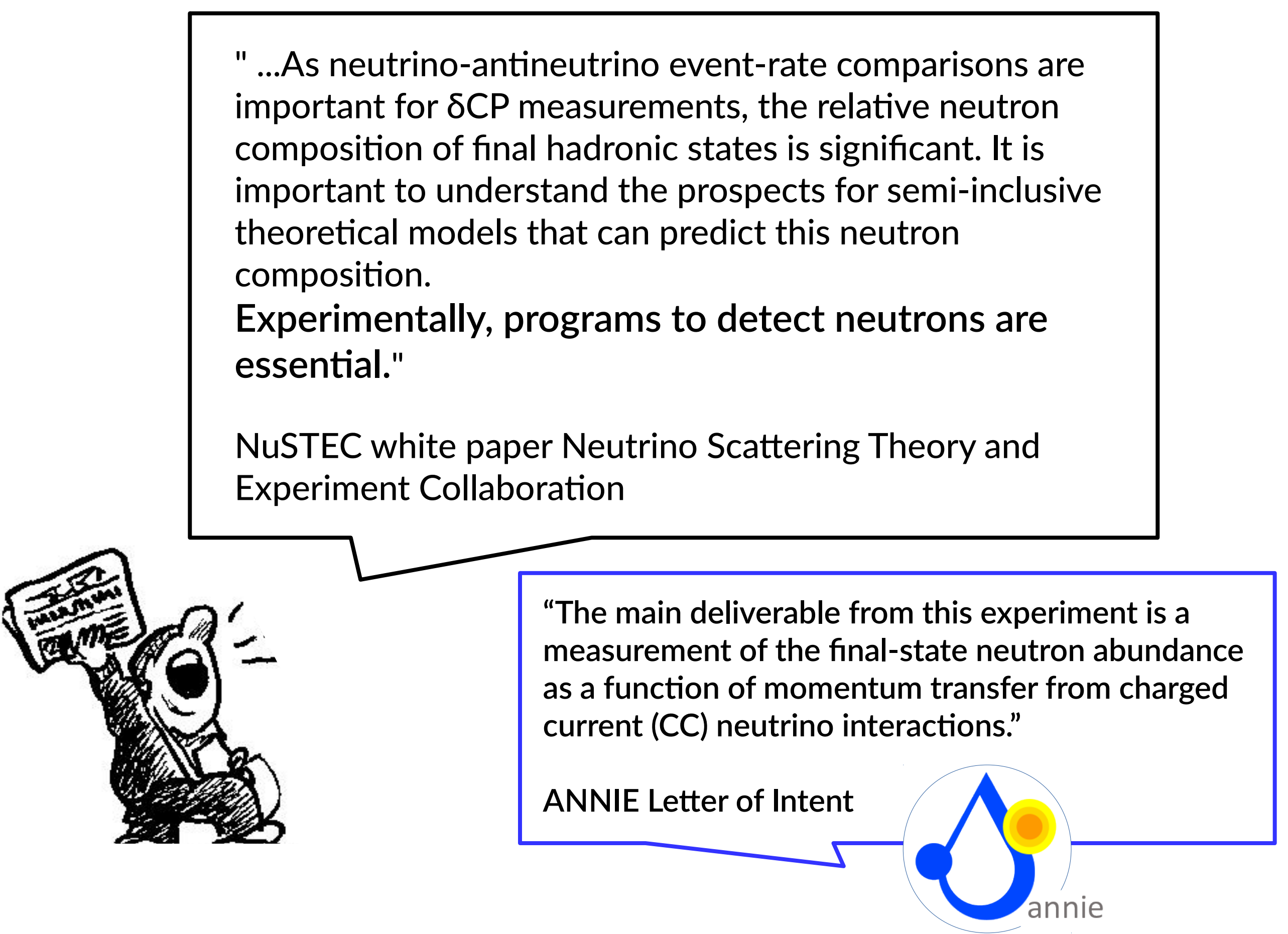




\section{Making Neutrons Visible}

- ANNIE will use Gadolinium doping to achieve neutron visibility

- 8MeV gamma cascade, $\sim 4-5 \mathrm{MeV}$ visible energy

- $20 \mu$ s capture time helps minimize backgrounds

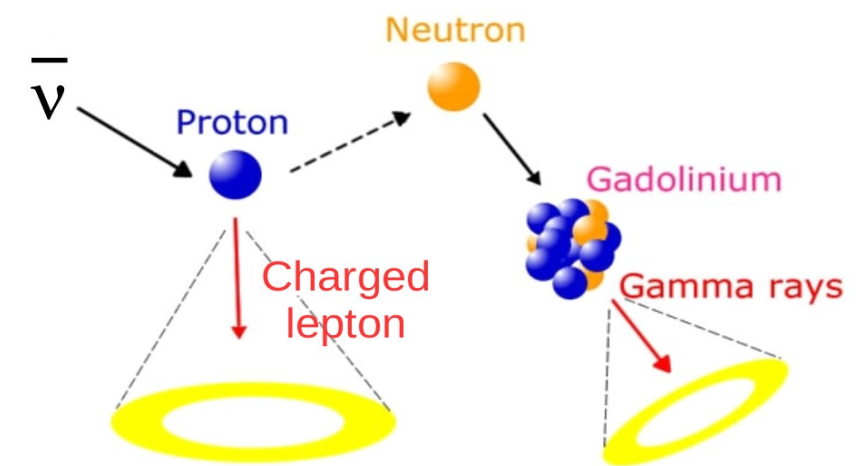

Originally detectable signal New signal

- $20 \mathrm{~cm}$ capture distance ensure neutrons do not leak out of the tank

- Nonetheless, given ANNIE's small size it's crucial to maximize fiducial volume

We need vertex resolution $\sim 10 \mathrm{~cm}$, or equivalently, timing resolution $\sim 100 p s . .$.

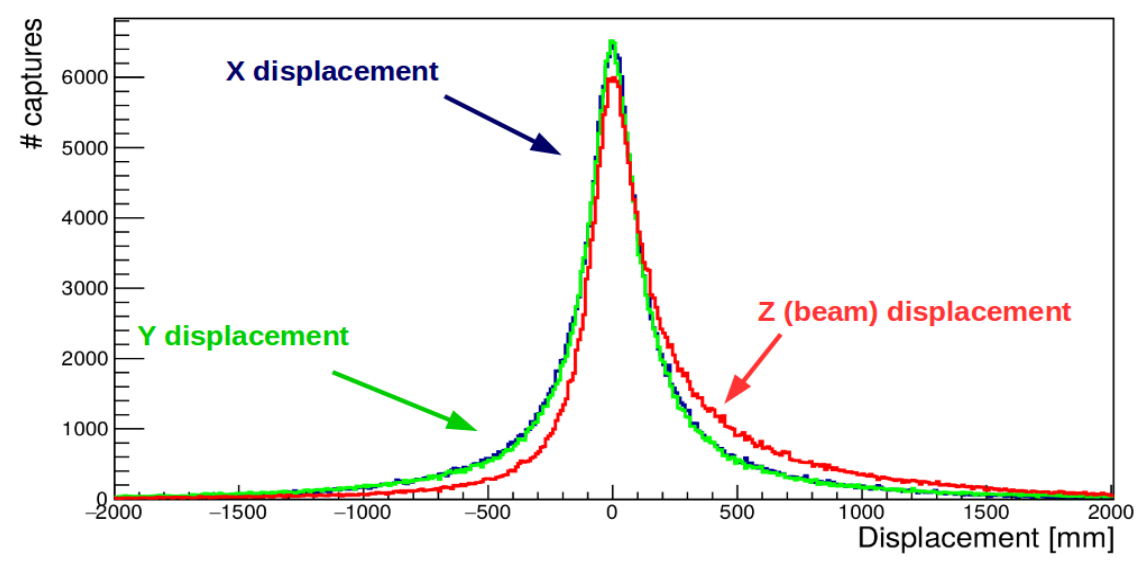




\section{A New Generation of Photodetector}

Large Area Picosecond Photodetectors (LAPPDs)

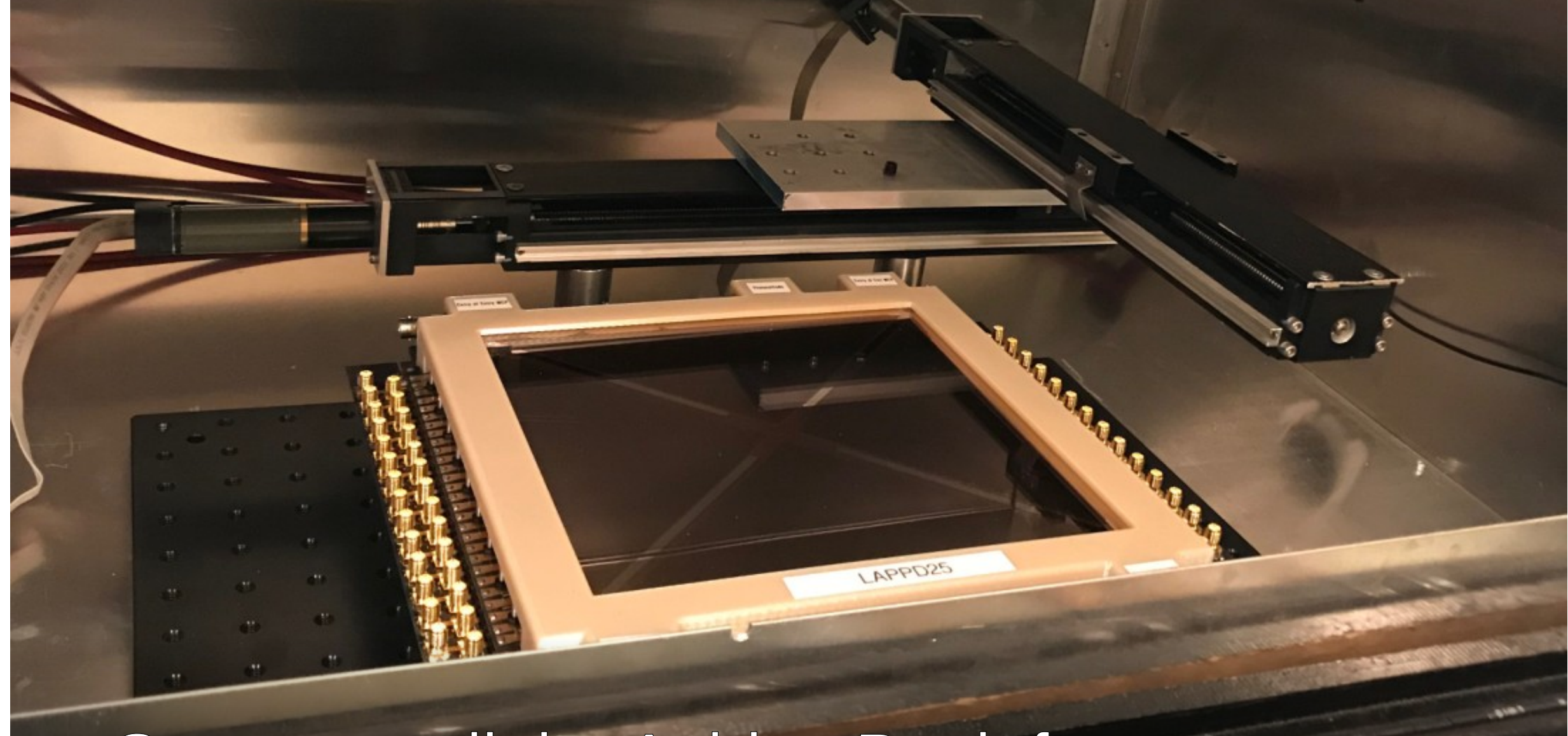

See next talk by Ashley Back for more... 


\section{The ANNIE Detector}

\section{Forward Veto Gd Doped Water Tank}

Muon Range Detector
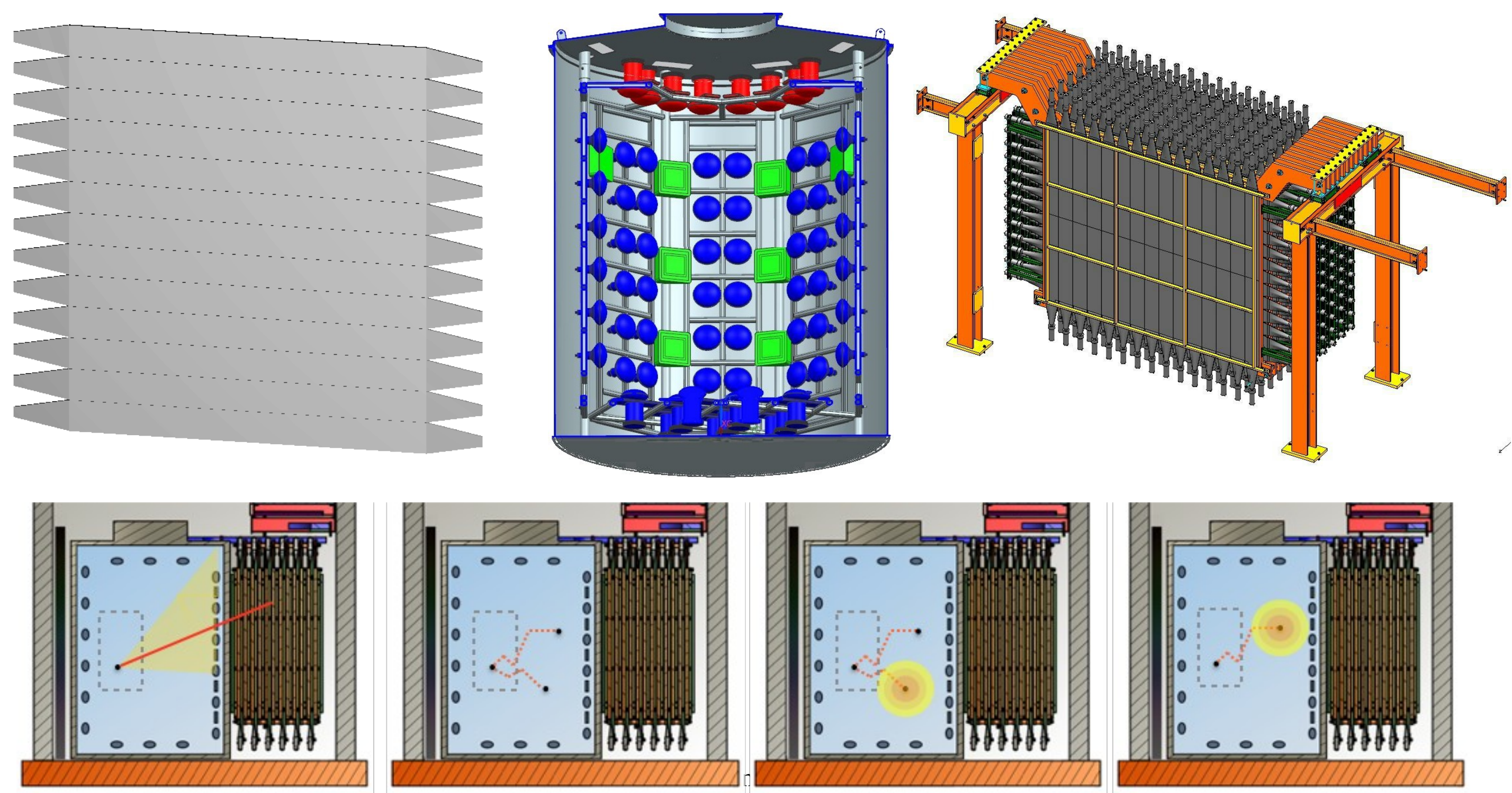


\section{ANNIE Phase 1}

- Aside from efficiency we also need to know backgrounds

- Primary sources are upstream dirt interactions and 'skyshine' neutrons, scattered from the atmosphere

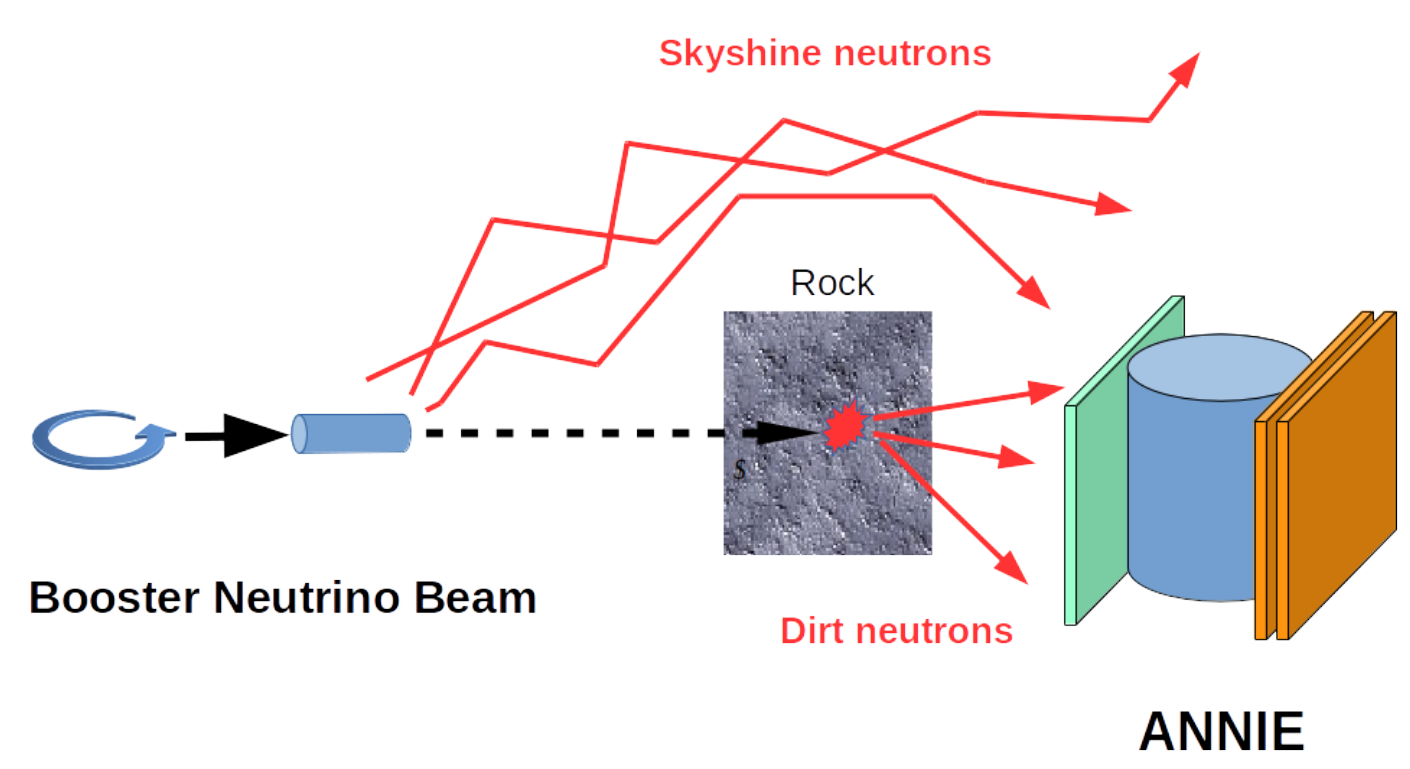

Image: Vincent Fischer

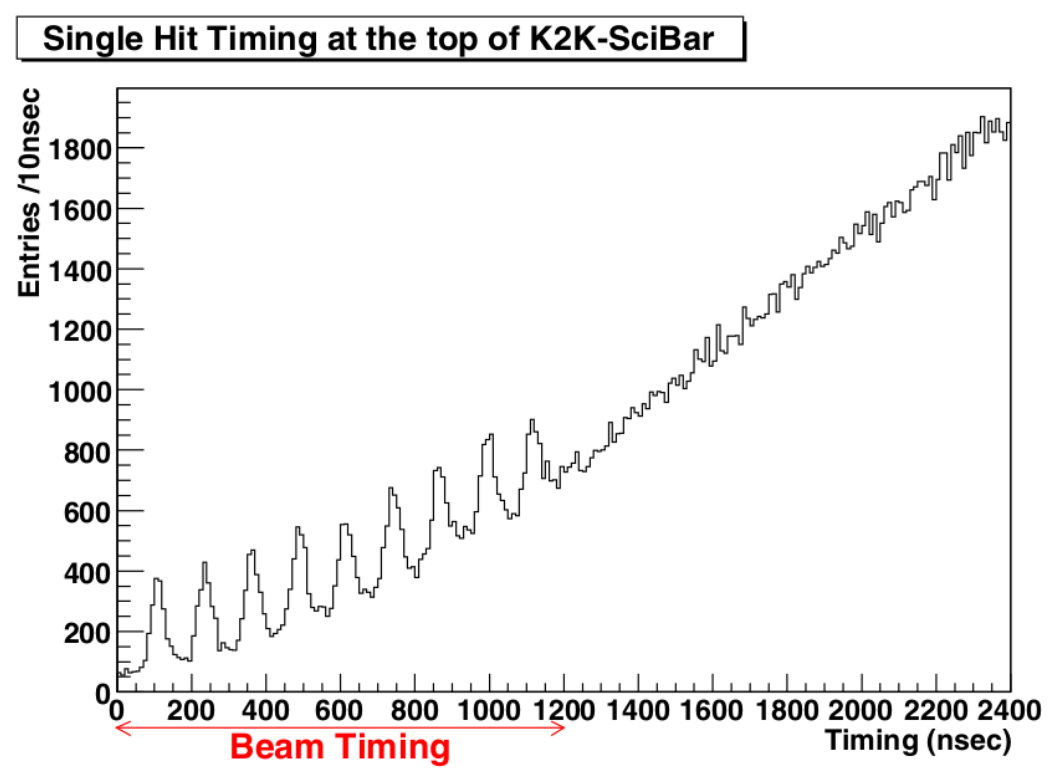

arXiv:hep-ex/0601022 


\section{Phase 1 Detector}

- Moveable sub-volume of liquid scintillator doped with $0.25 \% \mathrm{Gd}$

- Optically isolated from tank, with two PMTs watching for events

- White tank liner, 60 PMTs on the bottom to veto muons by cherenkov light

- Positional scan was performed to measure drop-off with overburden and distance from the beam-side wall

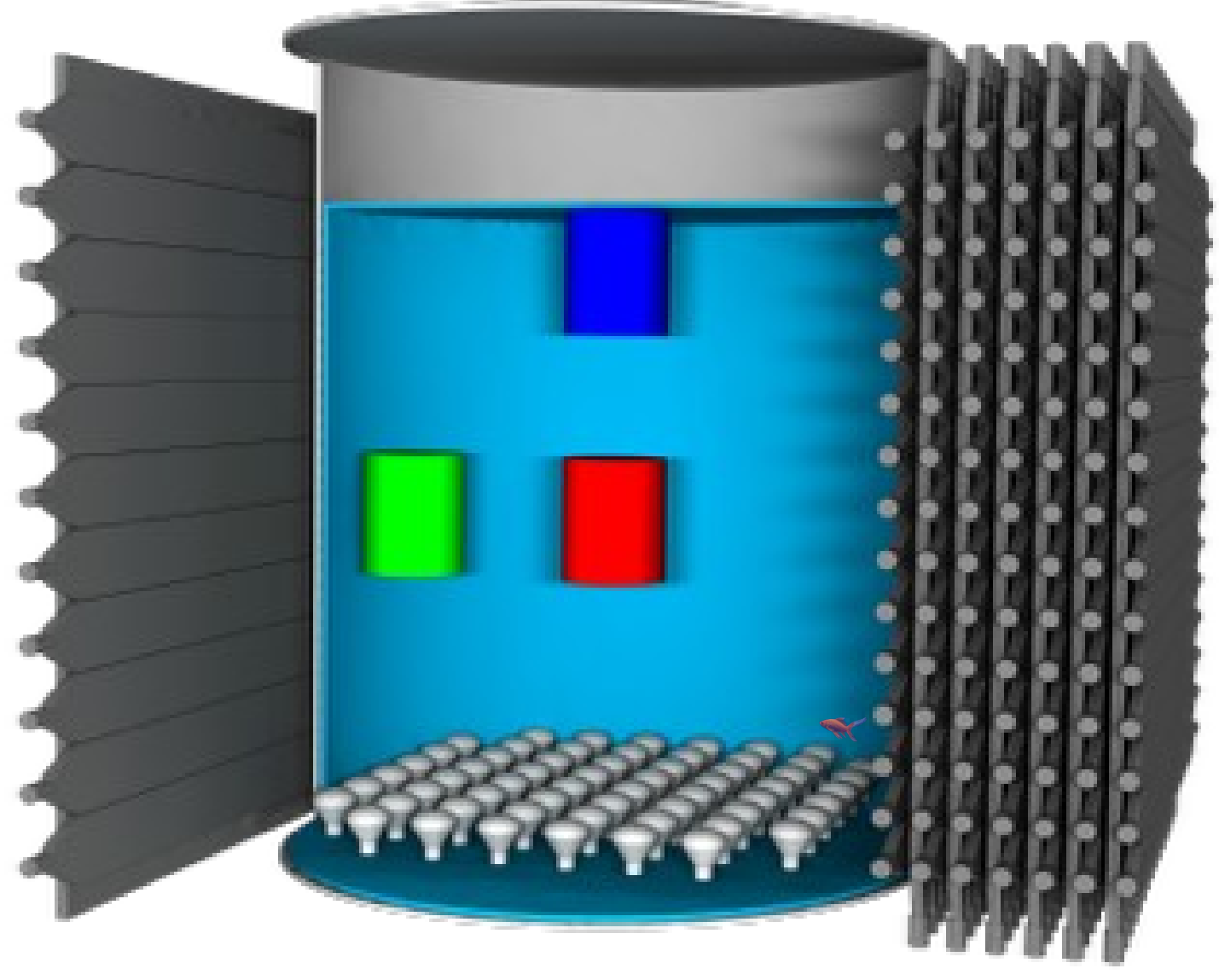

Credit: Jonathan Eisch 


\section{Detector Calibration}

- Neutron response was calibrated with a ${ }^{252} \mathrm{Cf}$ source, using a scintillator crystal to trigger on gammas emitted during fission

- Source activity was measured using a commercial neutron detector and another source of well-known activity

- Results show the expected $13 \mu$ s capture time and agree fairly well with Monte Carlo simulations

- Multiple methods of NCV efficiency calculation were used, with good agreement between them
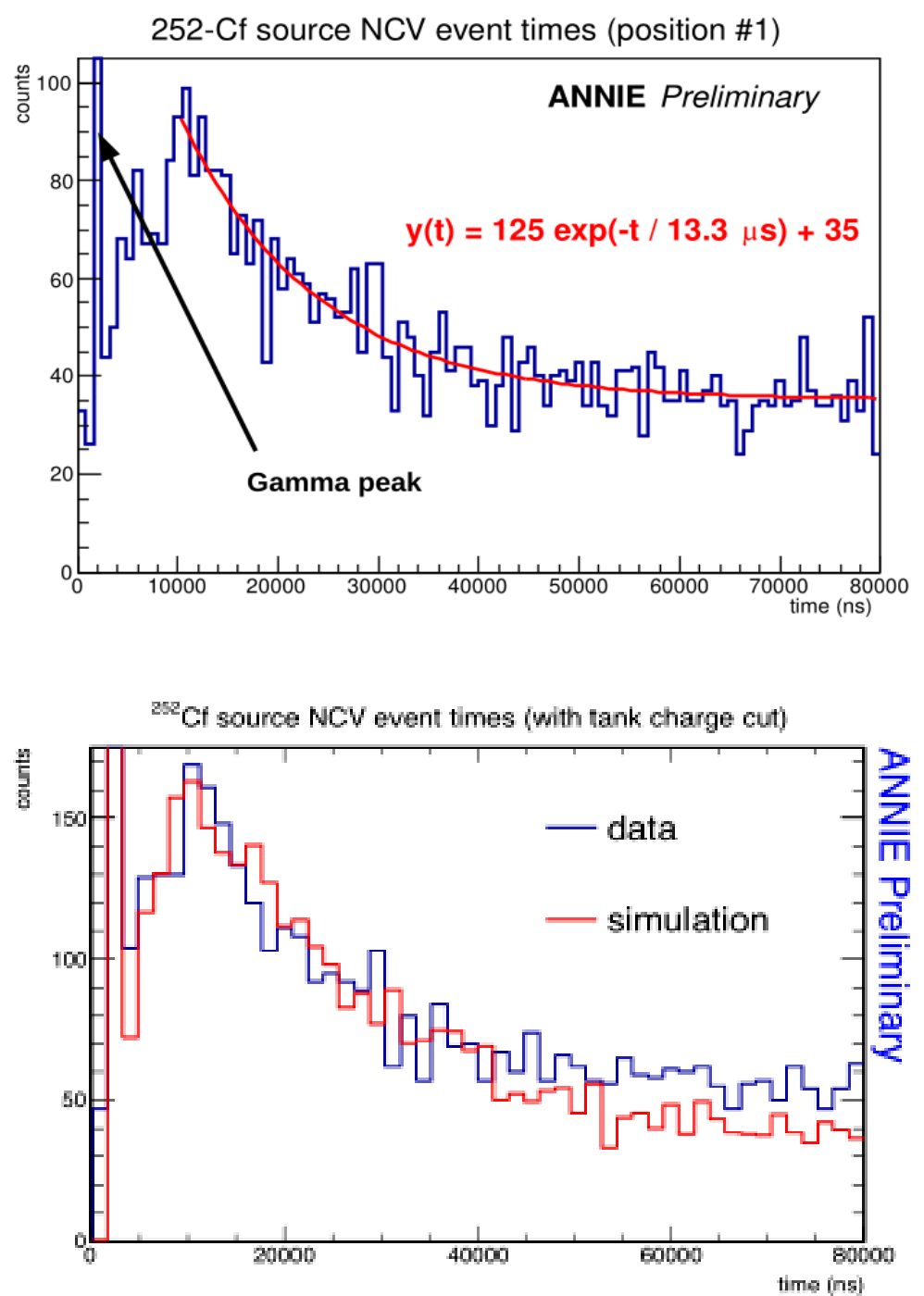


\section{Results}

- Shown is the time profile of events, averaged over many beam spills

- Events are defined as simultaneous hits on both NCV PMTs, with no corresponding tank event

- Position \#1 is at the water surface, Position \#2 is at the tank centre

- There is a peak of in-time events consistent with neutral current events and proton recoils from thermalizing neutrons

- Following this, there is a rise, then exponential decay of neutron capture events

- There is a stark reduction in neutron events at depths $>2 \mathrm{ft}$

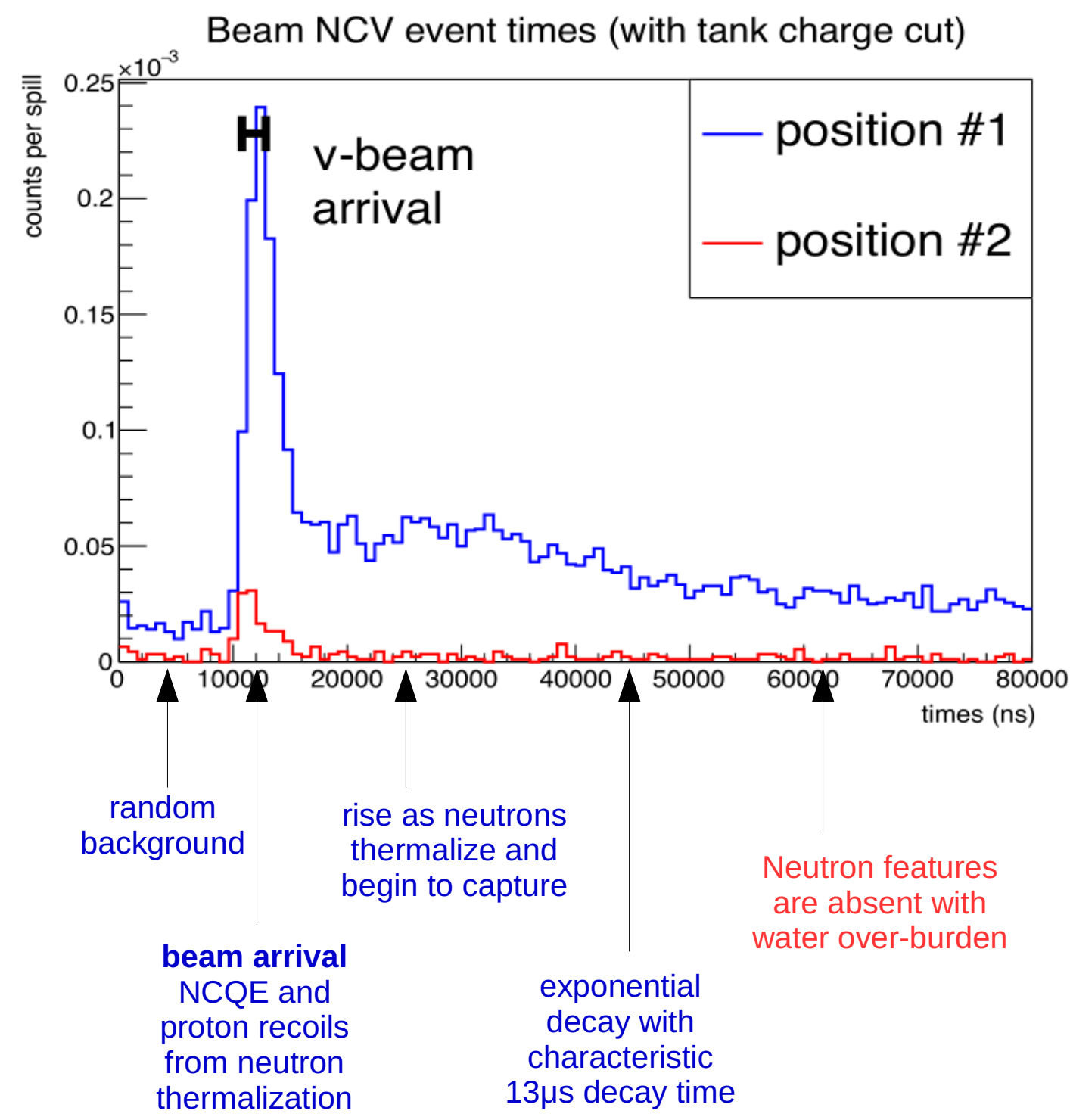




\section{Results}

- The integrated neutron capture counts for seven positions within the tank are shown

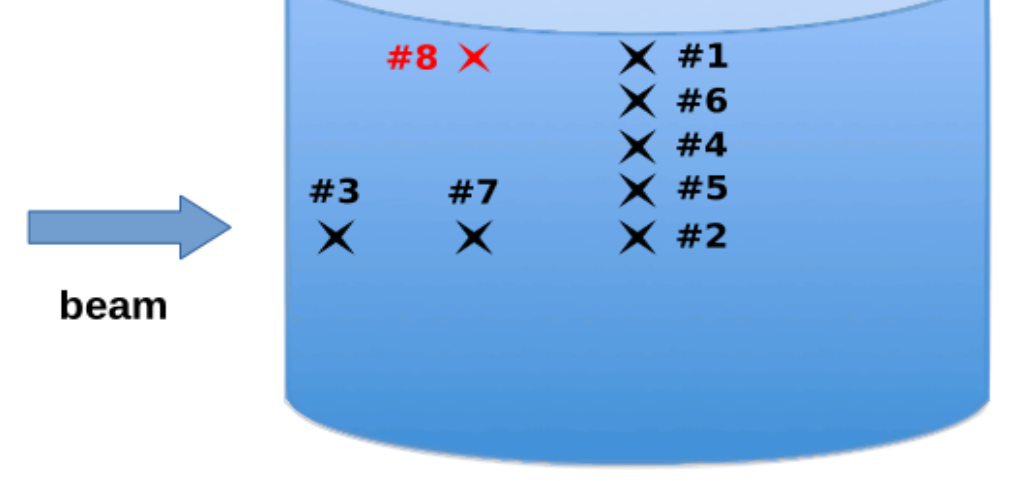

- The blue curve traces the drop-off of skyshine with increasing water overburden

- The red curve shows the drop-off of dirt events with increasing distance from the front wall

- Background neutron rates are less than 0.02 per spill per $\mathrm{m}^{3}$ at all locations with half a meter of water overburden

- Based on this, Phase II will use the full 2.5 ton fiducial volume with good detection efficiency

Neutron capture rate results

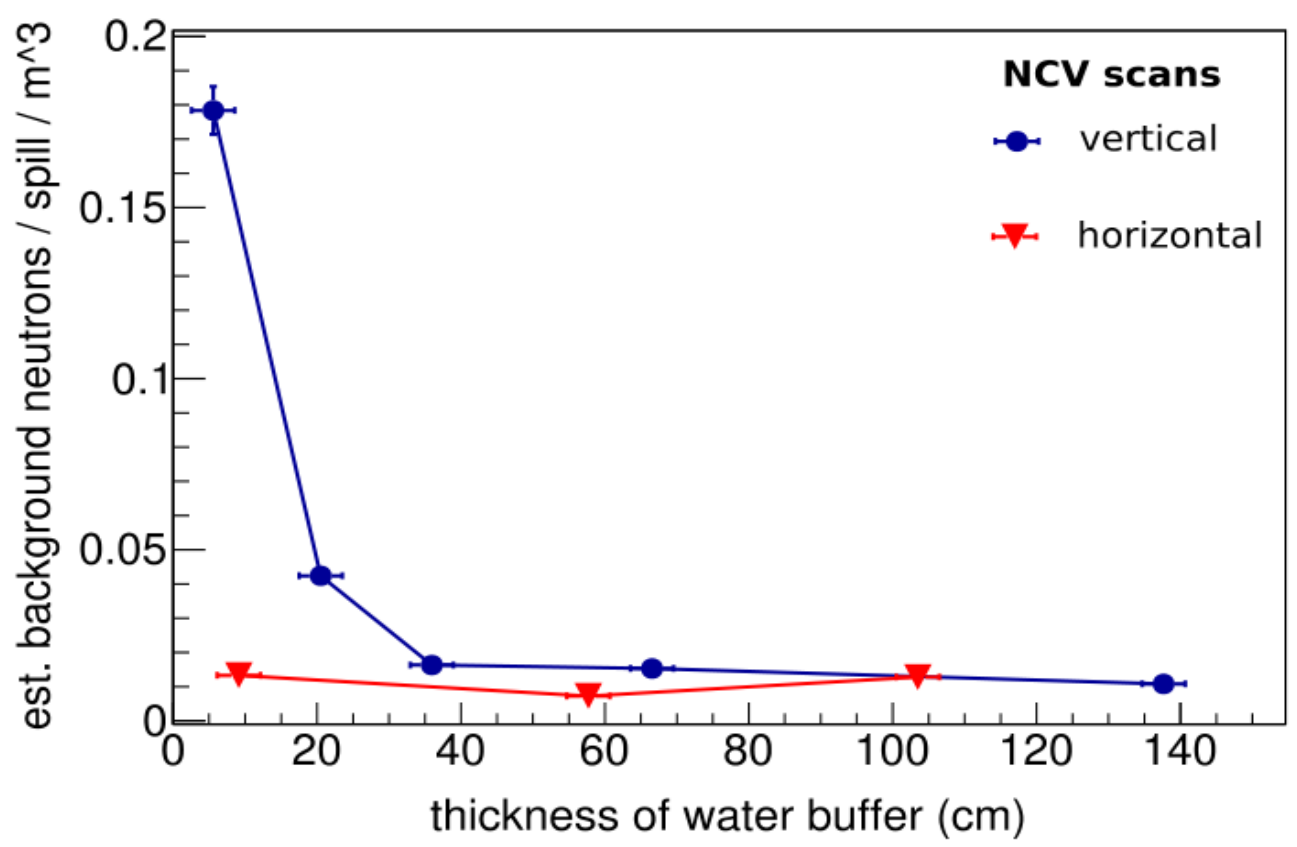

plot by Steven Gardiner 


\section{Toward ANNIE Phase II}

- Detector is being upgraded

- Gd compatibility testing is ongoing

- LAPPDs and electronics are being integrated and characterized

- Simulations and reconstruction are being heavily developed
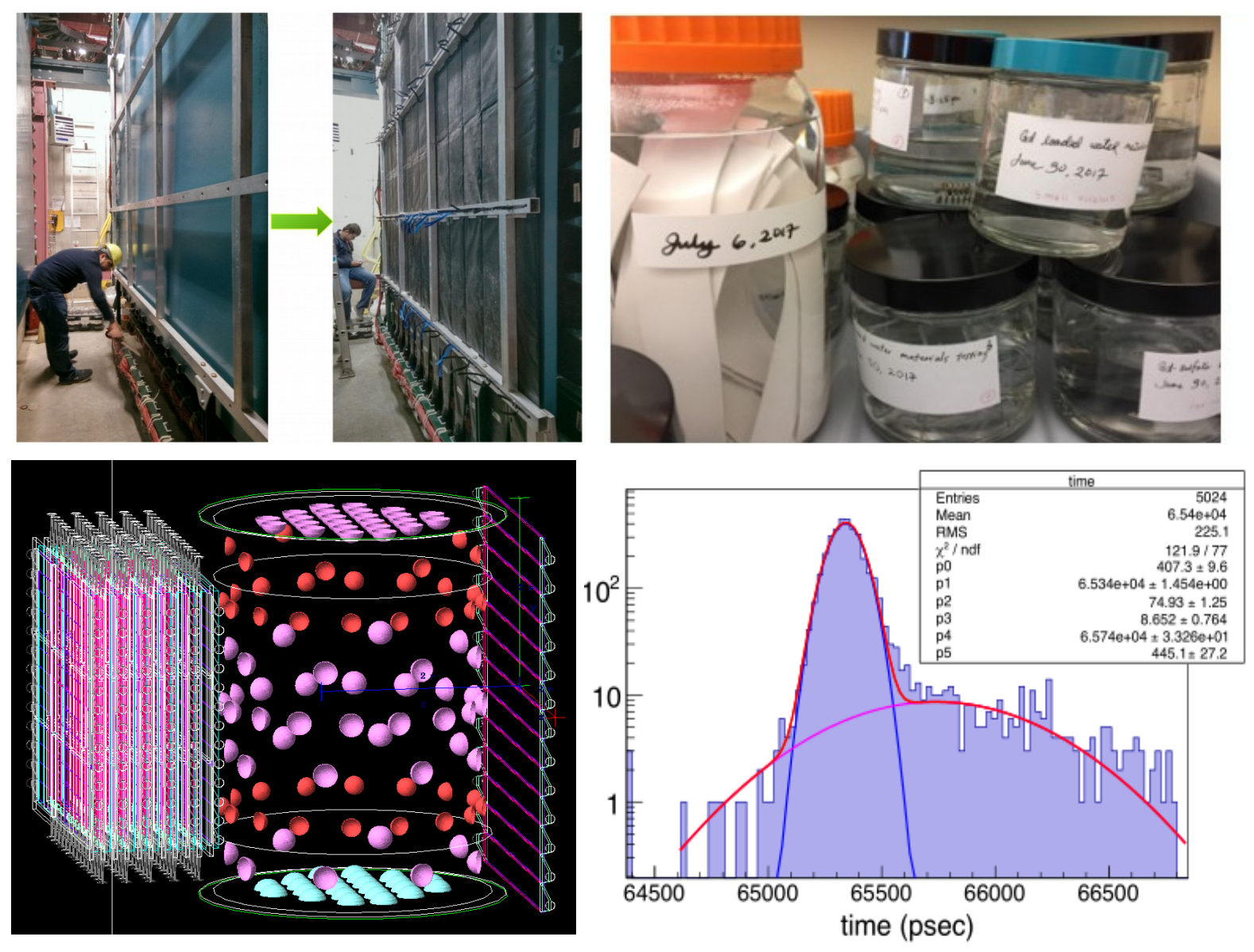


\section{Conclusions}

- ANNIE will measure neutron multiplicity, a key handle for testing interaction models of multi-nucleon final states

- Improve understanding of neutron tagging, with applications to background tagging in PDK and DNSB searches, reaction antineutrino detection ...

- Implement LAPPDs in a particle detector, gaining experience with a nextgeneration technology

- I presented the results from Phase I background measurement

- Low neutron backgrounds, suitable for physics

- Phase II preparation is progressing

- ANNIE will start taking physics data in 2018 


\section{Thank you for listening}

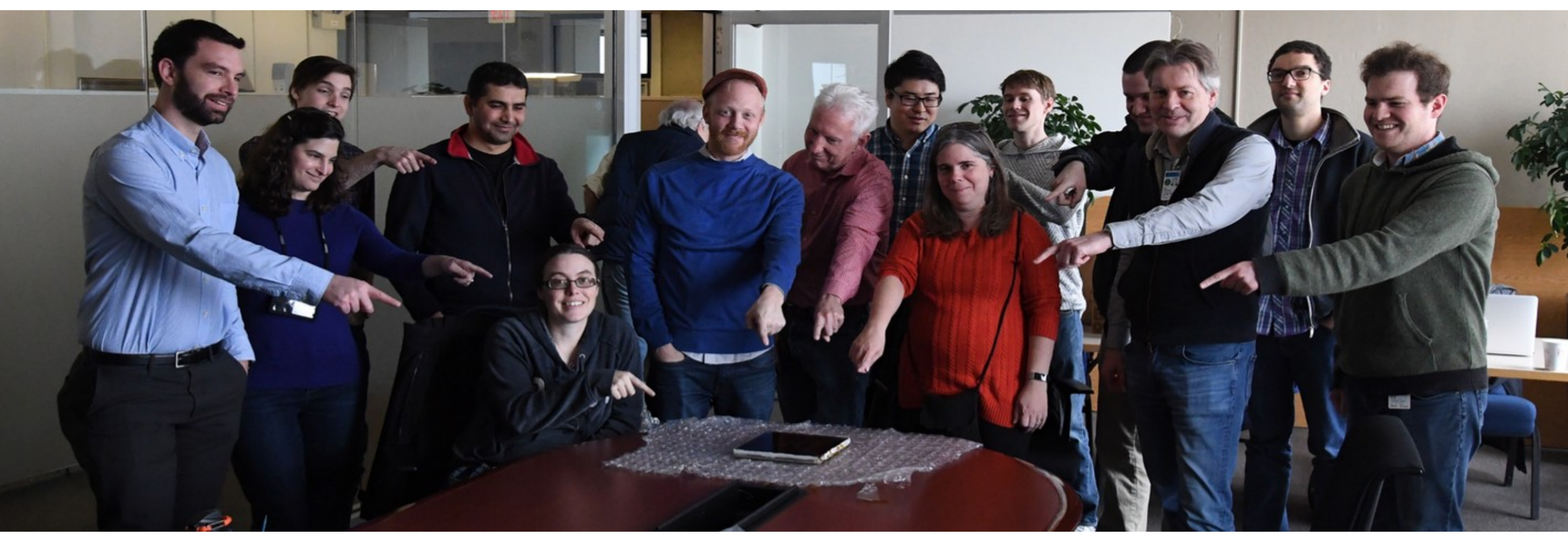

ANNIE Collaboration Meeting, January 2017 


\section{backup slides}




\section{Fake CCQE}

Many different modes, but the majority of them produce 1 or more neutrons

\begin{tabular}{|r|l|l|l|l|}
\hline Interaction Fraction & Inclusive & $\mathbf{0}$ Neutron Sample & $\mathbf{1}$ Neutron Sample & $\begin{array}{l}\text { More Than 1 Neutron } \\
\text { Sample }\end{array}$ \\
\hline Truth-level CCQE & $67.80 \%$ & $91.99 \%$ & $46.65 \%$ & $37.29 \%$ \\
\hline MEC & $20.45 \%$ & $4.44 \%$ & $37.37 \%$ & $37.44 \%$ \\
\hline Single Pion Prod. & $10.12 \%$ & $3.13 \%$ & $14.15 \%$ & $21.22 \%$ \\
\hline Deep Inelastic Sc. & $1.47 \%$ & $0.23 \%$ & $1.74 \%$ & $3.89 \%$ \\
\hline Tisc. Final State Int. & $0.16 \%$ & $0.21 \%$ & $0.09 \%$ & $0.16 \%$ \\
\hline
\end{tabular}

M. Wetstein, Talk at Fermilab Final State Nucleons Meeting

$$
4 \mathrm{GeV} \mathrm{e}^{-} \text {on }{ }^{12} \mathrm{C}
$$

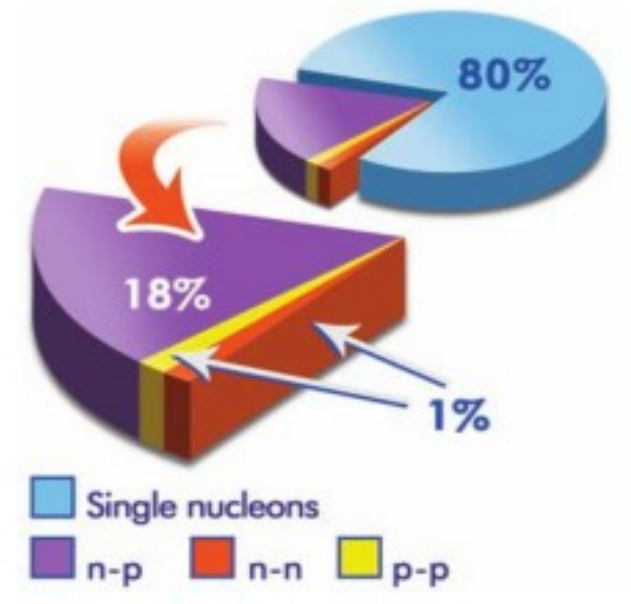

Reconstructed $\mathrm{E}_{v}$ assuming CCQE kinematics

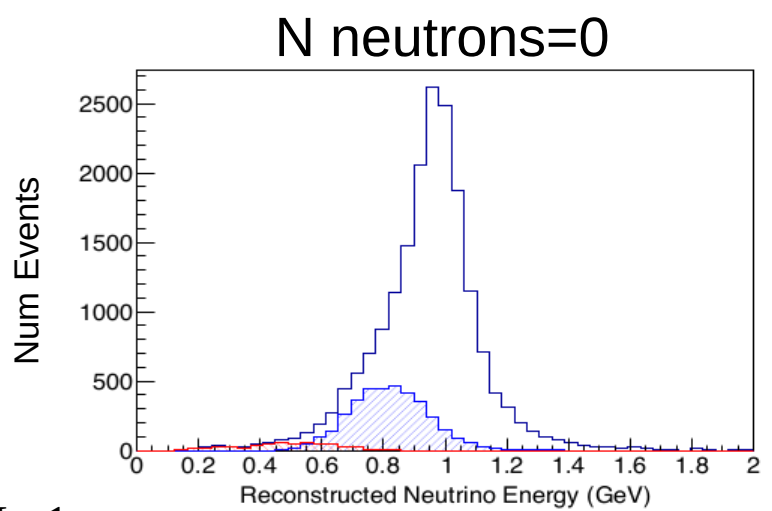

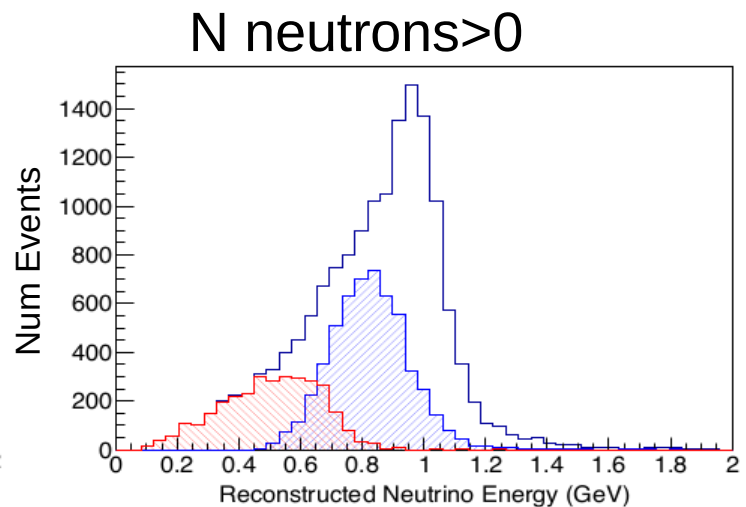

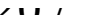




\section{Electronics Development}

- 4-channel 500MHz ADC boards developed for KOTO at the University of Chicago, built for ANNIE.

- These cards are used in a several thousand channel DAQ in KOTO.

- Continuous full-waveform digitization:

- pipeline self-triggering algorithm

- digital delay-line ring-buffer.

- Custom firmware developed at lowa State for recording prompt and delayed capture signals

- 16-channel board developed by University of Chicago also available.

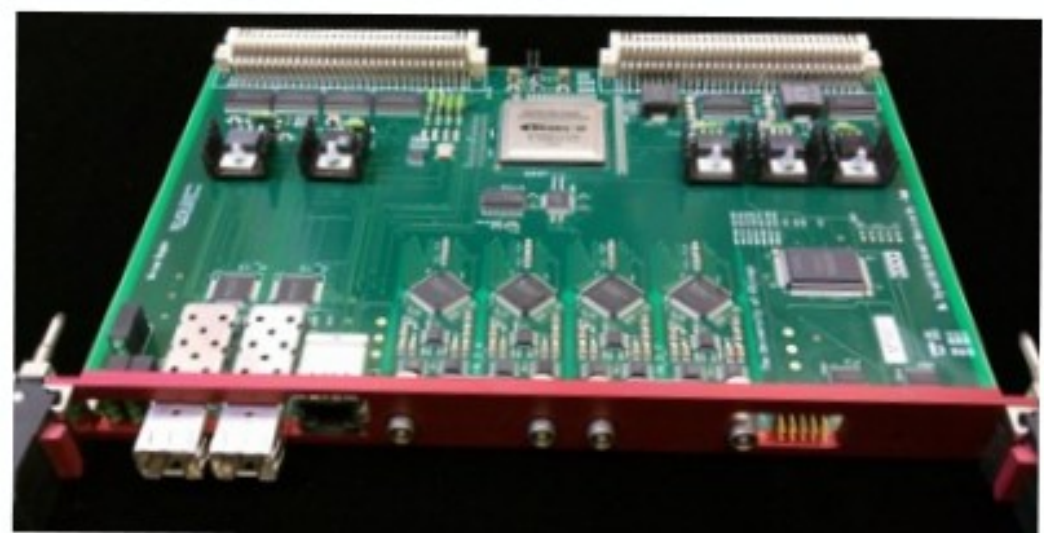

4-channel 500MHz ADC card

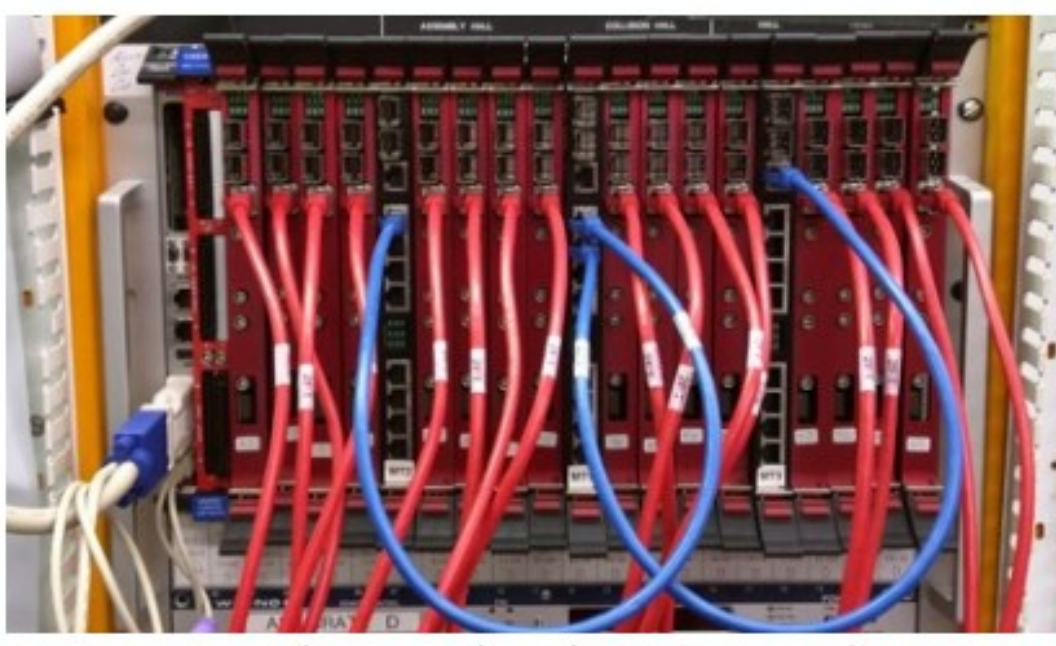

64-channel+trigger cards 


\section{Cross-Section Measurements}

- SciBooNE and T2K have made $v_{\mu}$ charged-current inclusive cross sections on carbon

- No similar measurement on water (oxygen) target exists. T2K recently made such a measurement (not yet published), but the result is flux-integrated

- ANNIE plans to measure differential CC-inclusive crosssection in first Phase II span, others with further running

- Complementary measurement on Ar by SBN-ND could allow ${ }^{18} \mathrm{Ar}:{ }^{16} \mathrm{O}$ ratio measurement with systematics cancellation 


\section{Possible Neutral Current Cross-section Measurement}

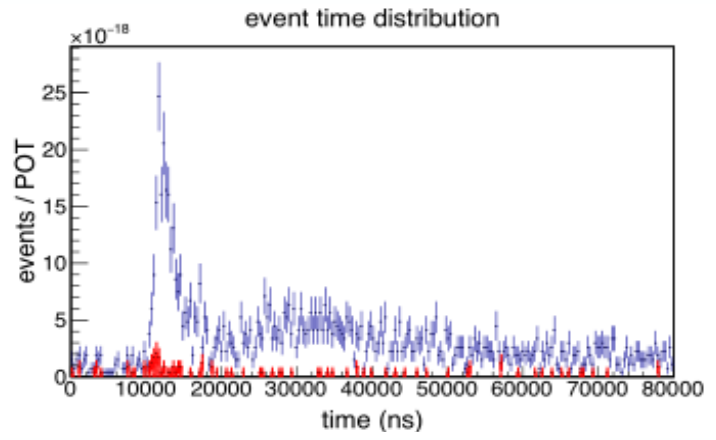

Irreducible excess of non-muon activity in coincidence with the beam seems to be consistent with two detectable neutral current signatures:

- proton recoils in the scintillator

- de-excitation gammas

\section{Tagging Nuclear De-excitation Gammas}

- Neutrino interactions typically leave the nucleus in an excited final state

- Many de-excitations involve the emission of MeV gammas with an $\mathrm{O}(1)$ sec time constant

- There is an opportunity to expand on prior work looking at de-excitations from neutral current interactions (T2K/Super-K)

- There is an opportunity to demonstrate a newer capability: tagging de-excitation gammas following a CC-interaction. This would provide a handle to separate between CC interactions on $\mathrm{H}$ versus $\mathrm{O}$

Neutral currents with a prompt gamma and subsequent neutron capture are a background for IBD neutrino interactions.

ANNIE can characterize and measure those backgrounds

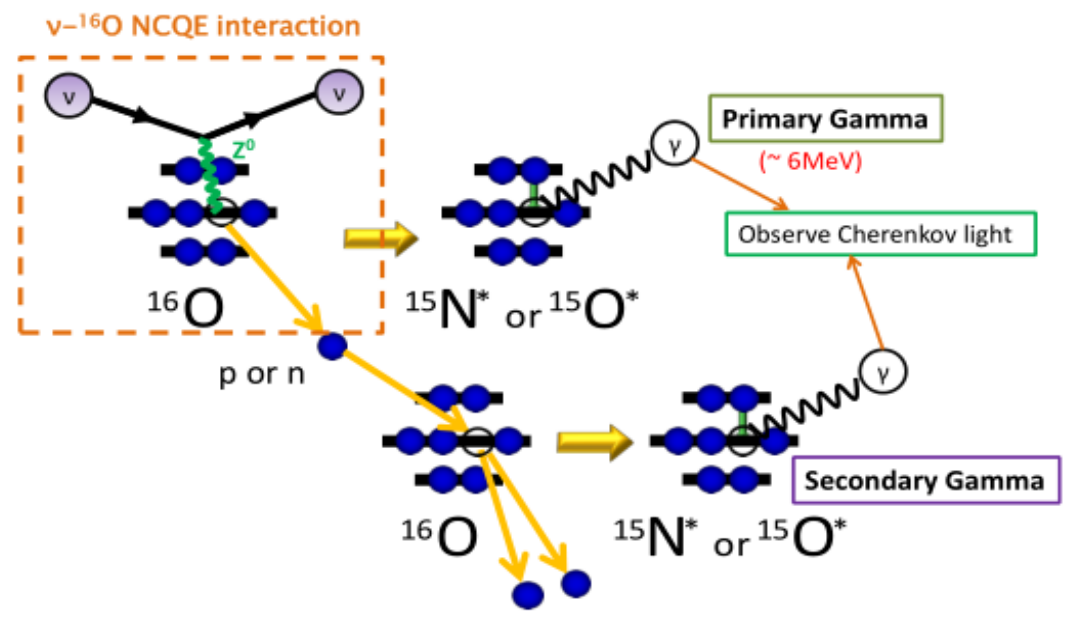

source: dissertation of Huan Kuxian (T2K) 


\section{Neutron Acceptance and Efficiency}

- BNB energy is $\sim 0.7 \mathrm{GeV}$, maximum neutron energy is $\sim$ few hundred MeV

- Capture distance is < tank radius, even for highest energy neutrons

- Effectively no upper bound on energy than the beam energy

- Although $10 \%$ of neutrons $>10 \mathrm{MeV}$ are expected to capture on Oxygen!

- No lower energy bound as neutrons stop then capture

- Efficiency is therefore just matter of detection, not retention, and is $\sim 50-60 \%$

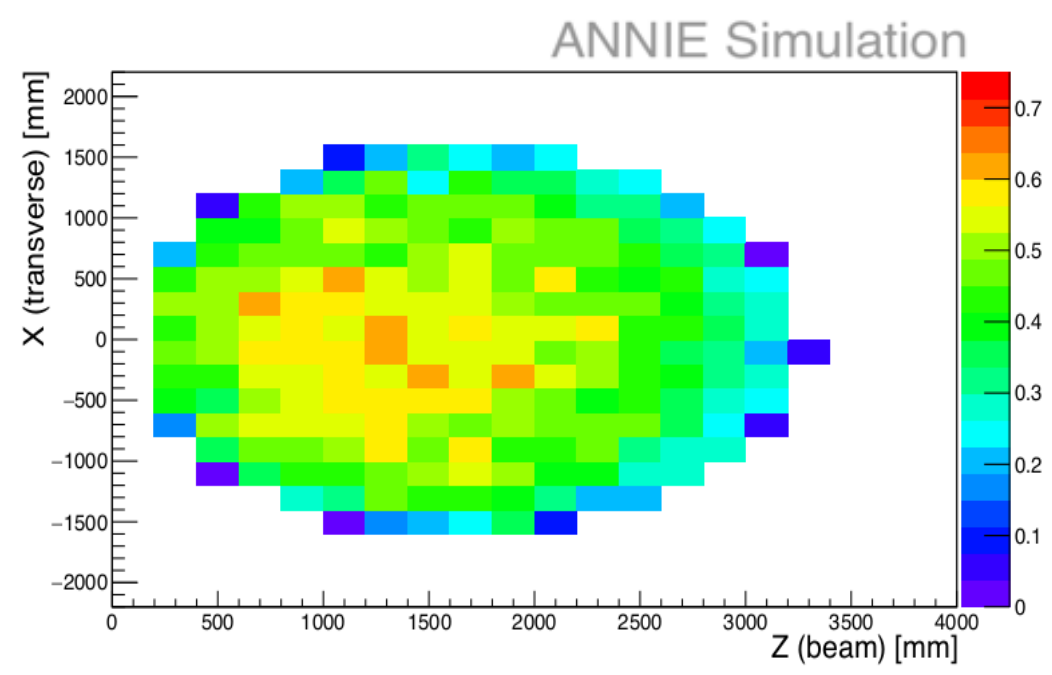

Neutron detection efficiency vs neutrino

interaction position within $2 \mathrm{~m}$ fiducial y axis

region, 10pe threshold

V. Fischer, U.C. Davis 


\section{NCV Efficiency: Method 1}

- Scale MC to data, extract efficiency from best fit

- Had difficulty with RAT-PAC neutron simulations being out by factor 100 - fixed by writing interface to FREYA

- Remaining difficulty that source composition not well known, unknown pile-up contribution from $n$ and gammas

- Low stats, poor signal to noise

- Gives $\sim 9 \%$ efficiency - slightly lower than cosmic muon derived detection threshold 


\section{NCV Efficiency: Method 2}

- Cosmic trigger structure used to select muons passing through NCV

- Plenty of data

- Steps:

- Plot distribution of total charge on both NCV PMTs in cosmic muon events in which both NCV PMTs fired (position \#2)

- Plot distribution of energy deposition in NCV for simulated cosmic events

- Compare peaks $\rightarrow$ energy to charge scaling factor (assume linear)

- We trigger on ADC counts, not total NCV charge, so make histogram of total NCV charge for events in which both PMTs fired at minimum threshold

- Find mean. This is the total NCV charge we're effectively triggering on.

- Convert this charge to energy with above.

- Simulate thermal neutron captures.

- Determine fraction of captures with energy deposition above threshold $=$ fraction of detectable neutron captures, or efficiency

- Gives $\sim 12 \%$ efficiency 


\section{NCV efficiency: Method 3}

- Count integrated neutron candidates in window after gamma peak

- Subtract estimated backgrounds:

- Constant in time, estimated from before-beam

- Gamma Pileup = fission rate * window time * count in (gamma peak / source triggers)

- Beam accidentals

- Compare to MC expected signal $\rightarrow$ Efficiency

- Expected source events = \#triggers * prob capture in NCV within time window * NCV efficiency

- Expected beam accidentals $=($ fission rate $*$ window time $) *$ prob capture in NCV * NCV efficiency 


\section{NCV Efficiency: Method 3}

- Binned likelihood fit to source run time profile

- $-9 \%$ efficiency, robust to parameter variation

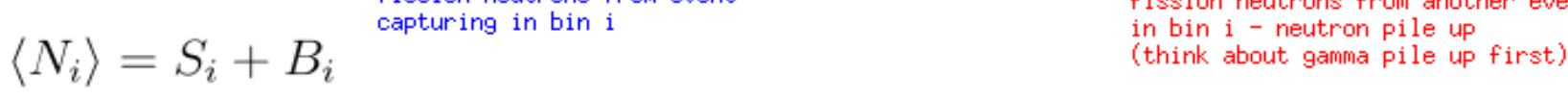

$$
\begin{aligned}
& =N_{\text {trigs }} \epsilon_{\mathrm{NCV}} \stackrel{P(\mathrm{n} \text { capture in } i \text { th bin|fission }}{\Delta t_{\mathrm{i}} R_{\text {fission }} P(\mathrm{n} \text { capture fission })} \\
& +N_{\text {trigs }} \Delta t_{i} \underset{R_{\text {fission }} P(\gamma \text { event|fission })}{\left.R_{\text {other }}\right)}+\delta_{i, \text { gamma bin }} N_{\text {trigs }} P(\gamma \text { event|fission }) \\
& \begin{aligned}
& \text { gamma pile up }= \text { random other events that trigger NCV } \\
& \text { number of fiste of random events } * \text { live time }
\end{aligned}
\end{aligned}
$$

- Expected bin counts are computed using the formula above

- Neutron capture probabilities taken from MC ( $10 \%$ statistical error on bin-wise values)

- 4 fit parameters: $R_{\text {fission, }} R_{\text {other }}, \epsilon_{\mathrm{NCV}}, P(\gamma$ event|fission $)$

- Vincent's measurement constrains fission rate

- Poisson distribution used to compute likelihood 


\section{Detecting Neutrons}

- ANNIE will use Gadolinium doping to achieve neutron visibility

- 49,700 bn neutron capture cross-section, averaged over natural isotopes

- $0.2 \% \mathrm{Gd}_{2}\left(\mathrm{SO}_{4}\right)_{3}$ doping results in $90 \%$ neutron captures on $\mathrm{Gd}$

- $8 \mathrm{MeV}$ total released energy, $\sim 4-5 \mathrm{MeV}$ visible energy

- as well reduce time window to reduce backgrounds.

- Want to be first experiment to do this first time

- pics of past failures

- compatibility testing

- Water filtration system

- Will be useful for

- helping tag antineutrino events too, for e.g. reactor /antiproliferation monitoring, contamination removal

- Identify CCQE vs CCQE-like

- Identify wrong-sign contamination

- Identify PDK / DSNB backgrounds 


\section{Data Recording}

- High efficiency 'Hefty' mode: 2us windows each time there is one of multiple trigger sources

- RWM

- Npmts

- Cosmic ray tagger

- Either NCV PMT

- Software: also drives LED

- Source trigger from LySO crystal

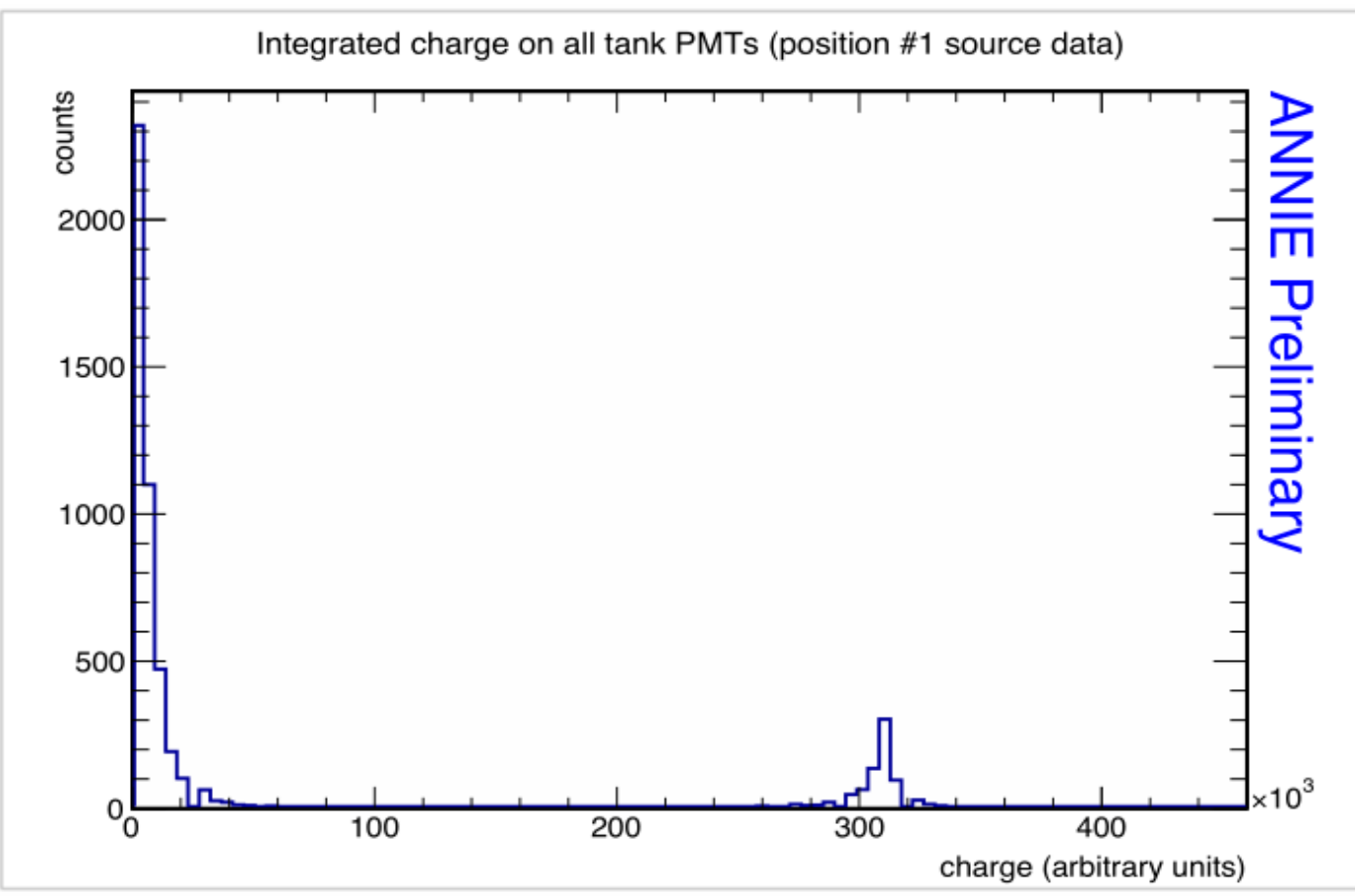

- Random, zero-bias

- Also read out any TDC hits from MRD within 80us from trigger 


\section{Event Rates}

- BNB delivers $4 \times 10^{12}$ POT per $1.6 \mu$ S spill at $5 \mathrm{~Hz}$

- Mean energy $0.7 \mathrm{GeV}$

- 93\% pure $v_{\mu}, 6.4 \% \bar{v}_{\mu}, 0.6 \% v_{\mathrm{e}}$ and $\bar{v}_{\mathrm{e}}$

- Average $1 \mathrm{CC} v_{\mu}$ interaction every 150 spills; no pileup

Event counts in a 2.5-ton fiducial volume over $2 \times 10^{20}$

POTs, or 1 year of running

All

Entering MRD

Stopping in MRD

$\begin{array}{llll}\text { NC } & \text { CC } & \text { CCQE } & \text { CC-Other } \\ 11323 & 26239 & 13674 & 12565 \\ 2 & 7466 & 4279 & 3187 \\ 2 & 4830 & 2792 & 2038\end{array}$




\section{Source Calibration}

- Cf-252 source with a measured activity of $9927 \pm 1275 \mathrm{n} / \mathrm{s}$

- LYSO (Lutetium Yttrium Oxyorthosilicate) crystal coupled to a 1-inch PMT used as a fission trigger

- PMT sees scintillation light from gamma-rays emitted during fission; all neutron producing fissions also produce gammas

- Gamma-rays also generate events in the NCV, visible in the first microseconds of the event time distributions

- After $\sim 10 \mu$ s thermalization time, neutrons capture with a time constant of $\sim 13 \mu \mathrm{s}$

- Efficiency is extracted from event time-profile using a binned likelihood method 